\title{
A special and rare case with osteomyelitis: A case report
}

\author{
Fuyong Jiao, Xianpeng Yan* \\ Department of Pediatrics, Shaanxi Province People’s Hospital, Xi’an, China \\ Email: *Yxp_9021@163.com
}

Received 28 May 2013; revised 30 June 2013; accepted 10 July 2013

Copyright (c) 2013 Fuyong Jiao, Xianpeng Yan. This is an open access article distributed under the Creative Commons Attribution License, which permits unrestricted use, distribution, and reproduction in any medium, provided the original work is properly cited.

\begin{abstract}
Background: Neonatal acute hematogenous osteomyelitis is very rare in clinical research. Early diagnosis and treatment to reduce the sequelae is very important. The purpose is to introduce a rare and special case to medical workers, so that readers know how to diagnose and treat such special cases. Methods: Through the clinical manifestations, imaging studies and the results in blood culture. Results: There were no adverse sequelae after effective antibiotics, which were discharged. Conclusion: There is great importance in early diagnosis and treatment of neonatal osteomyelitis.
\end{abstract}

Keywords: Newborn; Femur Distal in Left;

Osteomyelitis; Ceftriaxone Sodium

\section{INTRODUCTION}

Neonatal acute hematogenous osteomyelitis is very rare in clinical research, but it has a higher morbidity. Early diagnosis and treatment to reduce the sequelae is very important. Based on conservative therapy, due to high incidence of osteomyelitis and doctors' misdiagnosis, delayed treatment will cause the risk of myeloma, and it can lead to serious parlaysis of limbs, even endanger life security. Therefore, the correct osteomyelitis diagnosis can gain time, and the treatment of children will provide a reference for the relevant cases.

\section{CASE REPORTS}

Children, boy, 29 days, were admitted to hospital due to left knee swelling with activity restricted. 22 days ago (after seven-day birth) children for no apparent reason the left lower extremity knee swelling, no local skin redness, decrease in activity over the right side of the lower extremity, easy crying, no fever, and the spirit of the food can be satisfied, shot at a local hospital left knee radiograph: no abnormalities, give local hot symptomatic treat-

${ }^{*}$ Corresponding author. ment, not medication. 2 d check blood: 110.1 white blood cells $/ \mathrm{mm}^{3}$ (61.1\% neutrophils, 29.6\% lymphocytes), yet do special processing to continue local heat treatment, were not seriously ineffective. $6 \mathrm{~d}$ at a local hospital before admission, review radiographs of the left knee (Figure 1): left distal femur bone destruction and periosteal reaction, and give more consideration to the infection without treatment. Further treatment is conducted in our hospital.

Admission examination: Temperature: $36.8^{\circ} \mathrm{C}$, Pulse: 145 times/min, Breathing: 41 times/min, Weight: 4.5 kg, normal development, nutrition, moderate, conscious spirit of fair, body skin, mucous membranes without jaundice, rash and bleeding point, the anterior fontanelle $2.0 \mathrm{~cm} \times 2.0 \mathrm{~cm}$, soft, cardiopulmonary examination was normal, soft abdomen, liver and spleen is not next to no time, the umbilical skin congestion, the visible little yellow secretions, normal bowel sounds. Outreach of the left lower limb flexion, left knee mild swelling, skin redness, skin temperature is normal, no tenderness, activity slightly limited the right lower extremity normal. Admission diagnosis: left knee swelling reasons unknown origin: 1) the left distal femur osteomyelitis? 2) Sepsis? 3) malignant bone tumor? Omphalitis. Blood: 72.6 white blood cells $/ \mathrm{mm}^{3}$ (23.5\% neutrophils, 59\% lymphocytes), urine stool, CRP, anti-O were normal ESR $19 \mathrm{~mm} / \mathrm{h}$ after admission. The ceftriaxone sodium (T), 50 $\mathrm{mg} / \mathrm{kg}$ three weeks of treatment and supportive care, at the same time the energy left distal femur CT (Figure 2): the local destruction of bone lesions of the left distal femur see punctate high density lesions soft tissue swelling, consider the infection. The left distal femur MRI: abnormal signal intensity in the left femur metaphyseal and epiphyseal cartilage associated with soft tissue swelling, to consider infectious lesions may. The day of admission to do blood cultures, one week after the return: Staphylococcus aureus. Left lower extremity activity in children increased significantly after treatment, the swelling subsides. Review the blood after treatment of $10 \mathrm{~d}$, CRP, erythrocyte sedimentation rate were normal and a half 


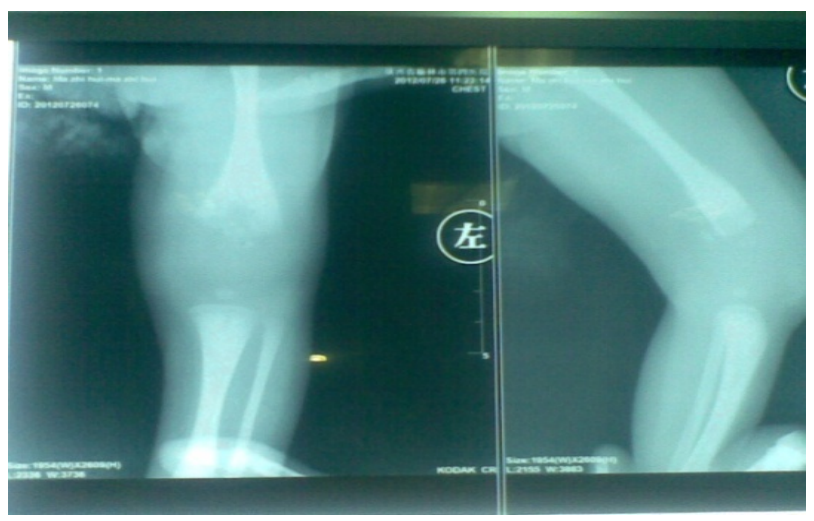

Figure 1. Before treatment.

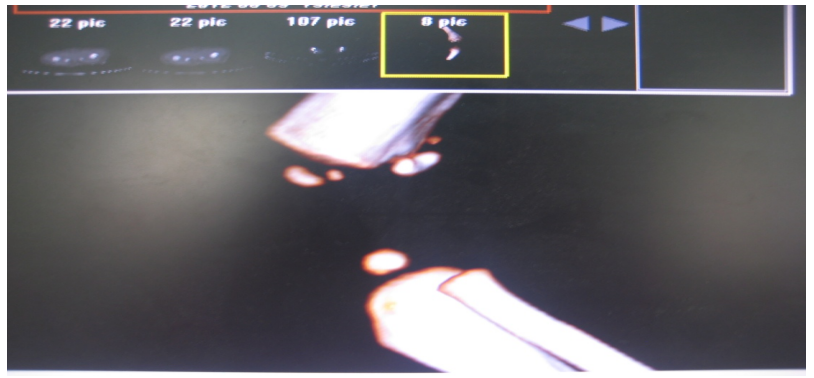

Figure 2. The treatment of the CT findings: the left distal femur local destruction of bone lesions see punctate high density lesions soft tissue swelling, consider infection.

months after the review of blood culture: negative. After three weeks of treatment, the children generally umbilical no redness, no abnormal secretions, the left knee no swelling, no tenderness, skin temperature and normal left lower extremity and the right lower extremity. Review of radiographs of the left knee (Figure 3): left distal femur see multiple cystic density to reduce the area, the edge see bone defect, and I was no exception to change. Followed for 2 months after discharge, children with movement normal development of limb length contralateral no difference, no angular deformity.

\section{DISCUSSION}

Acute osteomyelitis in neonatal period is rare, the incidence rate of the neonatal intensive care unit hospitalized children is $1 / 1000$ - 3/1000 [1], but the clinical manifestations, imaging and laboratory tests are not typical cases. Common causes and predisposing factors of blood-borne infections include umbilical arterial cannulation, heel puncture phlebotomy, as well as premature birth, respiratory distress syndrome invasive operation [2]. Infancy, bone blood supply than adults, rich, and metaphyseal rich capillary network, actively growing, and here slow blood flow and deposition of beneficial bacteria, stay and reproduction, growth. So, the blood-borne osteomyelitis occurrs in young people, even occurs in babies born

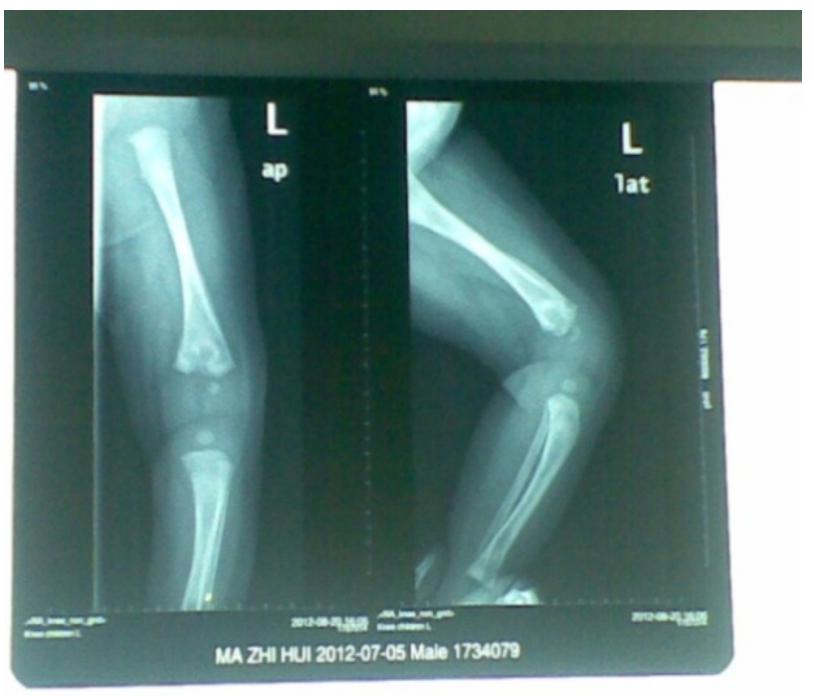

Figure 3. After 3 weeks of treatment.

dozens of days, and also affects many parts of the metaphysis of long bones. Osteomyelitis also decides on the strength of the human resistance. Such resistance includes systemic resistance and local resistance. The cause of osteomyelitis is coupled with diseases due to congenital deficiency in frail health, malnutrition, exposure to cold and other weakened body resistance factors. Low local resistance, pediatric skin, bone delicate skin barrier function, immune function are not perfect, the children are vulnerable to injuries from falls near the joint sprains, it makes small bleeding of metaphyseal ruptured capillary network, or nearby tissue and cell rupture, therefore, the bacteria easily stay breeding, and it may cause osteomyelitis occurrence. Osteomyelitis symptoms of blood infection in children, including the decrease in the affected limb movement, tenderness, swelling, common fever or no fever, often last for more than 2 weeks, infection often involves multiple bone adjacent joints and soft tissue. The cases consider umbilical infection, bacterial invasion, as the blood reaches the metaphysis of long bones incidence. The laboratory tests normal or elevated WBC, ESR, and CRP. About 14-day earlier films of swollen joints may be normal, prone to misdiagnosis, this is the case of children, the radiographs CT of left knee shows partial destruction of the periosteum, periosteal reaction after about 20 days, in line with osteomyelitis image manifestations. Synovial fluid and blood culture for etiological diagnosis is especially important, but at low positive rate [3]. The white blood cell is not a reliable indicator of osteomyelitis, the white blood cell in the presence of infection is still within the normal range [4]. ESR is elevated in the majority of cases, but its severity is not consistent with osteomyelitis. C-reactive protein as an inflammatory product, relatively speaking, is a reliable indicator, it began to increase in 
the hours after infection and returned to be normal within 1 week after the condition was well controlled [5]. X-ray found that bone destruction is often a certain lag for the infection appeared after 10 - 21 d typical performance [6], the CT findings of osteomyelitis include bone tissue and adjacent muscle, muscle gap or subcutaneous tissue swelling also forms a cyst-like cyst and subperiosteal hematoma in the soft tissue gas, the fat liquid plane and sinus [7]. MRI diagnosis of soft tissue contrast range in the diagnosis of osteomyelitis and soft tissue infections, it is clear that there is priority in the X-ray, CT. MRI allows early detection of lesions of bone tissue, it is more suitable for the early diagnosis. The radionuclide bone tissue scan sensitivity is a relatively high, early application to detect lesions [8]. Ultrasound purulent fluid or infection caused by bone abscess, and providers include puncture, aspiration, biopsy, diagnosis and treatment guidelines. The treatment of choice of the new penicillin III plus cefotaxime sodium, ceftriaxone, cefuroxime sodium [2], MRSA infection vancomycin or selection of clindamycin intravenous drug improved clinical symptoms, inflammatory markers returned to be normal in the course of 3 - 6 weeks. Joint cavity decompression, drainage of pus, supportive therapy is necessary. Osteomyelitis can affect the length of the long bone growth and generate angular deformity, as well as early surgical orthopedic deformity correction. The damage to the Ministry of metaphyseal against ossification started sooner, and then the postoperative bone growth will be smaller [9]. Osteomyelitis neonatal period is closely related to the prognosis, good diagnosis and effective anti-inflammatory treatment is important for atypical cases, early diagnosis is particularly important, it has direct impact on the growth, development and quality of life of children in the future of the bones. The effective anti-inflammatory treatment was conducted for 3 weeks, and then was discharged in March after the left knee radiographs were completely normal.

\section{REFERENCES}

[1] Korakaki, E., Aligizakis, A., Manoura, A., et al. (2007) Methicillin-resistant Staphylococcus aureus osteomyelitis and septic arthritis in neonates: Diagnosis and management. Japanese Journal of Infectious Diseases, 60, 129-131.

[2] Gutierrez, K. (2005) Bone and joint infections in children. Pediatric Clinics of North America, 52, 779-794. doi:10.1016/j.pcl.2005.02.005

[3] Qu, L., Xu, J.X. and Wen, J.D. (2012) Neonatal osteomyelitis in one case. Neonatology, 17, 39.

[4] Chen, D.X. (2012) Thin accounted for the status and progress of the east. Treatment of osteomyelitis. The Chinese Orthopaedic Surgery, 20, 224.

[5] Unkila-Kallio, L., Kallio, M.J., Eskola, J., et al. (1994) Serum C-reactive protein, erythrocyte sedimentation rate, and white blood cell count in acute hematogenous osteomyelitis of children. Pediatrics, 1, 59.

[6] Kaim, A.H., Gross, T. and von Schulthess, G.K. (2002) Imaging of chronic posttraumatic osteomyelitis. European Radiology, 5, 1193-1202. doi:10.1007/s00330-001-1141-0

[7] Hui, C.L. and Naidoo, P. (2003) Extramedullary fat fluid level on MRI as a specific sign for osteomyelitis. Australasian Radiology, 4, 443-446. doi:10.1046/j.1440-1673.2003.01217.x

[8] Level, X.F. (2010) The osteomyelitis imaging diagnosis. Chinese and Foreign Medical Research, 19, 91.

[9] Song, K.S. and Kim, H.K. (2005) Regeneration of the proximal tibial epiphysis after infantile osteomyelitis: Report of three cases with an 8- to 22-year follow-up. Journal of Bone and Joint Surgery, 87, 979-983. doi:10.1302/0301-620X.87B7.16027 